\title{
Transformation of the second order modulus by positive linear operators
}

\author{
Radu Păltănea and Gabriel Stan
}

\begin{abstract}
We obtain estimates for the transformation of the second order modulus of continuity by positive linear operators which satisfy certain conditions, which are common to a large class of approximation operators.
\end{abstract}

\section{Introduction}

The global smoothing preservation problem consists in the preservation of the Lipschitz classes by positive linear operators. Mention the earliest result of T. Lindvall [3]:

$$
B_{n}\left(\operatorname{Lip}_{1}(\alpha, M)\right) \subset \operatorname{Lip}_{1}(\alpha, M), n \in \mathbb{N}, 0<\alpha \leq 1,
$$

where $B_{n}$ are the classical Bernstein operators, given by

$$
B_{n}(f, x)=\sum_{k=0}^{n} f\left(\frac{k}{n}\right) p_{n, k}(x), p_{n, k}(x)=\left(\begin{array}{l}
n \\
k
\end{array}\right) x^{k}(1-x)^{n-k},
$$

$f \in B[0,1], x \in[0,1], n \in \mathbb{N}$ and $\operatorname{Lip}_{1}(\alpha, M)$ denotes the Lipschitz class of first order consisting in all functions $f$ which satisfy the inequality $\omega_{1}(f, \rho) \leq M \cdot \rho^{\alpha}$ for all $\rho>0$, with $M>0$ and $0<\alpha \leq 1$.

Key Words: global smoothness preservation, second order modulus of continuity, Mirakjan-Favard-Szász operators, Baskakov operators.

2010 Mathematics Subject Classification: 2010 Mathematics Subject Classification: 41A36, 41A17.

Received: 29 April, 2014.

Revised: 10 June, 2014.

Accepted: 30 June, 2014. 
On the other hand, D. X. Zhou [11] obtained that the Bernstein operators do not preserve the Lipschitz classes of the second order $\operatorname{Lip}_{2}(\alpha, M)$, where $\operatorname{Lip}_{2}(\alpha, M)$ denotes the set of all functions that satisfy the inequality $\omega_{2}(f, \rho) \leq M \cdot \rho^{\alpha}$ for all $\rho>0$, with $M>0$ and $0<\alpha \leq 2$. Recall that, the second order modulus is defined on an interval II by

$$
\omega_{2}(f, \rho)=\sup \{|f(x-h)-2 f(x)+f(x+h)|: x \pm h \in \mathbb{I}, 0<h \leq \rho\},
$$

where $\rho>0$ and $f: \mathbb{I} \rightarrow \mathbb{R}$ is such that the supremum above is finite.

In connection with the problem of global smoothness preservation is the problem of the transformation of the second order modulus by positive linear operators $L$ defined on $\mathbb{I}$ in the following form:

$$
\omega_{2}(L(f), \rho) \leq c \cdot \omega_{2}(f, \rho), f \in C(\mathbb{I}), \rho>0,
$$

where $c>0$ is a constant independent with regard to $f$ and $\rho$. This problem has sense only for the operators $L$ which preserve linear functions. In this direction a first result was obtained by C. Cottin and H. Gonska [2], who showed that for $c=4.5$ we have

$$
\omega_{2}\left(B_{n}(f), \rho\right) \leq c \cdot \omega_{2}(f, \rho), f \in C[0,1], \rho>0, n \in \mathbb{N} .
$$

Later on J. Adell and A. Pérez-Palomares [1] improved this result by showing that we may replace the constant $c$ in front of $\omega_{2}(f, h)$ by $c=4$. In the paper Păltănea [7] it is proved the following double inequality

$$
2 \leq \sup _{n \in \mathbb{N}} \sup _{f \in C[0,1] \backslash \Pi_{1}} \sup _{\rho \in\left(0, \frac{1}{2}\right]} \frac{\omega_{2}\left(B_{n}(f), \rho\right)}{\omega_{2}(f, \rho)} \leq 3 .
$$

The aim of the present paper is to obtain results for a larger class of operators, following the same ideas given in paper [7]. Then we give applications to several kinds of operators.

The second author used in [4] the technique of semi-groups of operators in an applicative problem.

\section{General results}

First we introduced the following notations:

For $A, B \subset \mathbb{R}$ we write $A<B$ if we have $a<b$, for all $a \in A$ and $b \in B$.

Let $\mathbb{I}$ be a real interval. We denote by $e_{j}(t)=t^{j}, t \in \mathbb{I}, j=0,1,2 \ldots$, the monomial functions.

If $\rho>0$, we denote $\rho \preceq \frac{1}{2}$ length(I), if $\rho \leq \frac{1}{2}$ length(I) in the case when interval $\mathbb{I}$ is closed and $\rho<\frac{1}{2}$ length $(\mathbb{I})$ in the case when interval $\mathbb{I}$ is not closed. 
Let $0<\rho \preceq \frac{1}{2}$ length $(\mathbb{I})$. We denote

$$
\begin{aligned}
\Gamma & :=\{(x, h) \in \mathbb{I} \times(0, \infty): x \pm h \in \mathbb{I}\} \\
\Gamma_{\rho} & :=\{(x, h) \in \Gamma, h \leq \rho\} .
\end{aligned}
$$

For any function $f: \mathbb{I} \rightarrow \mathbb{R}$, and $(x, h) \in \Gamma$ we denote

$$
\Delta_{h}^{2} f(x):=f(x+h)-2 f(x)+f(x-h) .
$$

Now we introduced a class of positive linear operators. Let $\Lambda$ be a finite or countable set. Let $\left(t_{l}\right)_{l \in \Lambda}$ be a set of knots $t_{l} \in \mathbb{I}, l \in \Lambda$. Let $\left(q_{l}\right)_{l \in \Lambda}$ be a set of continuous and positive functions on $\mathbb{I}$ which satify the following conditions

$$
\sum_{l \in \Lambda} q_{l}(x)=1, \quad \text { and } \quad \sum_{l \in \Lambda} t_{l} q_{l}(x)=x, \text { for all } x \in \mathbb{I} .
$$

Define a positive linear operator $L: V \rightarrow C(\mathbb{R})$, by

$$
L(f)(x):=\sum_{l \in \Lambda} q_{l}(x) f\left(t_{l}\right), f \in V, x \in \mathbb{I},
$$

where $V \subset C(\mathbb{I})$ is the linear subspace on which operator $L$ exists. Suppose that $e_{2} \in V$.

Lemma 2.1. Let operator $L$ defined above. For $(x, h) \in \Gamma$ and for all $l \in \Lambda$ denote $c_{l}:=c_{l}(x, h):=q_{l}(x+h)-2 q_{l}(x)+q_{l}(x-h)$.

Suppose that the set $\Lambda$ can be decomposed $\Lambda=I \cup J \cup K$, with $I<J<K$, such that

$$
c_{i} \geq 0,(i \in I), \quad c_{j}<0,(j \in J), \quad c_{k} \geq 0,(k \in K) .
$$

Then there exist the positive linear functionals $G_{j}: V \rightarrow \mathbb{R}, j \in J$, with the following properties:

$$
\begin{gathered}
\Delta_{h}^{2} L(f)(x)=\sum_{j \in J}\left(-c_{j}\right)\left[G_{j}(f)-f\left(t_{j}\right)\right], \quad f \in V . \\
G_{j}\left(e_{0}\right)=1, \quad \text { and } \quad G_{j}\left(e_{1}\right)=t_{j}, \text { for all } j \in J .
\end{gathered}
$$

Proof. We have

$$
\Delta_{h}^{2} L(f)(x)=\sum_{l \in \Lambda} c_{l} f\left(t_{l}\right), \text { for all } f \in V .
$$


From conditions (5) it follows that operator $L$ preserves linear functions. Consequently we obtain

$$
\sum_{l \in \Lambda} c_{l}=0, \quad \text { and } \quad \sum_{l \in \Lambda} t_{l} c_{l}=0
$$

Define

$$
\Delta:=\sum_{i \in I} c_{i} \cdot \sum_{k \in K} t_{k} c_{k}-\sum_{i \in I} t_{i} c_{i} \cdot \sum_{k \in K} c_{k}
$$

Since

$$
\begin{aligned}
& \sum_{i \in I} t_{i} c_{i} \cdot \sum_{k \in K} c_{k} \leq(\sup I) \sum_{i \in I} c_{i} \cdot \sum_{k \in K} c_{k} \\
& <(\inf K) \sum_{i \in I} c_{i} \cdot \sum_{k \in K} c_{k} \leq \sum_{i \in I} c_{i} \cdot \sum_{k \in K} t_{k} c_{k}
\end{aligned}
$$

it follows that $\Delta>0$.

For any $j \in J$ denote

$$
u_{j}:=\frac{1}{\Delta} \sum_{k \in K}\left(t_{k}-t_{j}\right) c_{k}, \quad \text { and } \quad v_{j}:=\frac{1}{\Delta} \sum_{i \in I}\left(t_{j}-t_{i}\right) c_{i}
$$

and consider the linear positive functional $G_{j}: C[0,1] \rightarrow \mathbb{R}$, given by

$$
G_{j}(f):=u_{j} \sum_{i \in I} c_{i} \cdot f\left(t_{i}\right)+v_{j} \sum_{k \in K} c_{k} \cdot f\left(t_{k}\right), \quad f \in C(\mathbb{I}) .
$$

It is a simple matter of calculation to derive relations (8) and (9) from relations (10), (11), (12), (13) and (14).

We need the following estimate with optimal constants, given in Păltănea [6], or [8], which we cite using the present notation.

Theorem $\mathbf{A}$ Let $\mathbb{I}$ be an arbitary interval and let $V \subset C(\mathbb{I})$ be a linear subspace such that $e_{j} \in V$, for $j=0,1,2$. Let $x \in \mathbb{I}$ and let $F: V \rightarrow \mathbb{R}$ be a positive linear functional with the property $F\left(e_{0}\right)=1$ and $F\left(e_{1}\right)=x$. Then we have

$$
|F(f)-f(x)| \leq\left(1+\frac{1}{2 \rho^{2}} F\left(\left(e_{1}-x e_{0}\right)^{2}\right)\right) \omega_{2}(f, \rho),
$$

for any $f \in V$, any $x \in \mathbb{I}$ and any $0<\rho \preceq \frac{1}{2}$ length $(\mathbb{I})$.

In this theorem it is admitted the possibility that $\omega_{2}(f, \rho)=+\infty$.

The main result is the following: 
Theorem 2.2. Let $L$ be a positive linear operator defined by (6) with conditions (5). Suppose that for each $(x, h) \in \Gamma$, if we denote $c_{l}:=c_{l}(x, h):=$ $q_{l}(x+h)-2 q_{l}(x)+q_{l}(x-h), l \in \Lambda$, then there are the sets $I, J, K$, depending on $x$ and $h$, such that $\Lambda=I \cup J \cup K, I<J<K$ and relation (7) fulfills. Suppose that there is also a constant $b>0$, such that

$$
\frac{\left|\Delta_{h}^{2} L\left(e_{2}\right)(x)\right|}{2 h^{2}} \leq b, \text { for any }(x, h) \in \Gamma .
$$

Then, for any $f \in V$ and $0<\rho \preceq \frac{1}{2}$ length $(\mathbb{I})$ such that $\omega_{2}(f, \rho)<\infty$, we have

$$
\omega_{2}(L(f), \rho) \leq(2+b) \omega_{2}(f, \rho) .
$$

Morover, if we have

$$
\lim _{h \rightarrow 0+} \sum_{l \in \Lambda}\left|\Delta_{h}^{2} q_{l}(x)\right|=0, \text { uniformly with regard to } x \in \operatorname{Int}(\mathbb{I})
$$

and $f$ is not a linear function, then

$$
\limsup _{\rho \rightarrow 0+} \frac{\omega_{2}(L(f), \rho)}{\omega_{2}(f, \rho)} \leq b .
$$

Proof. Let $0<\rho \preceq \frac{1}{2}$ length $(\mathbb{I})$. Let $0<h \leq \rho$ and choose $x$ such that $x \pm h \in \mathbb{I}$. Then we apply Lemma 2.1 and we obtain the positive linear functionals $G_{j}$, $j \in J$ which satisfies conditions (8) and (9). From (8) and Theorem A we have for $f \in V$ :

$$
\begin{aligned}
\left|\Delta_{h}^{2} L(f)(x)\right| & \leq \sum_{j \in J}\left(-c_{j}\right)\left|G_{j}(f)-f\left(t_{j}\right)\right| \\
& \leq \sum_{j \in J}\left(-c_{j}\right)\left[1+\frac{1}{2 h^{2}} G_{j}\left(\left(e_{1}-t_{j} e_{0}\right)^{2}\right)\right] \omega_{2}(f, h) .
\end{aligned}
$$

Taking into account that functionals $G_{j}$ preserve linear functions and aplying again relation (8) we obtain:

$$
\begin{aligned}
\sum_{j \in J}\left(-c_{j}\right) \frac{1}{2 h^{2}} G_{j}\left(\left(e_{1}-t_{j} e_{0}\right)^{2}\right) & =\frac{1}{2 h^{2}} \sum_{j \in J}\left(-c_{j}\right)\left[G_{j}\left(e_{2}\right)\left(t_{j}\right)-e_{2}\left(t_{j}\right)\right] \\
& =\frac{1}{2 h^{2}} \Delta_{h}^{2} L\left(e_{2}\right)(x) \\
& \leq b .
\end{aligned}
$$

Hence it follows that

$$
\frac{\left|\Delta_{h}^{2} L(f)(x)\right|}{\omega_{2}(f, \rho)} \leq \sum_{j \in J}\left(-c_{j}\right)+b, \text { for all }(x, h) \in \Gamma_{\rho} .
$$


If we pass to supremum with regard to $(x, h) \in \Gamma_{\rho}$, we obtain

$$
\frac{\omega_{2}(L(f), \rho)}{\omega_{2}(f, \rho)} \leq \sup _{(x, h) \in \Gamma_{\rho}} \sum_{j \in J}\left(-c_{j}\right)+b .
$$

Now, if we take into account that $-c_{j}=-q_{j}(x+h)+2 q_{j}(x)-q_{j}(x-h) \leq$ $2 q_{j}(x)$ it follows

$$
\sum_{j \in J}\left(-c_{j}\right) \leq 2 \sum_{j \in J} q_{j}(x) \leq 2 \sum_{j \in \Lambda} q_{l}(x)=2 L\left(e_{0}\right)(x)=2
$$

and combining with relation (20) it follows (17).

On the other hand, from (18) and (20) we obtain also (19).

\section{Applications}

\section{Mirakjan-Favard-Szász operators}

The Mirakjan-Favard-Szász operators are given by

$$
S_{n}(f)(x)=\sum_{k=0}^{\infty} s_{n, k}(x) f\left(\frac{k}{n}\right), n \in \mathbb{N}, x \in[0, \infty),
$$

where

$$
s_{n, k}(x)=e^{-n x} \frac{(n x)^{k}}{k !}
$$

and $f:[0, \infty) \rightarrow \mathbb{R}$ is such that series (21) converges.

The operators $S_{n}$ preserve linear functions. Let show that $S_{n}$ satify condition (7) for each $x \in(0, \infty), h>0$, such that $x-h \geq 0$, with certain sets $I<J<K$.

For $p \in[0,1]$ and $q \in[0, \infty)$,we consider the function

$$
\psi(t):=e^{-q}(1+p)^{t}+e^{q}(1-p)^{t}-2, t \in[0, \infty) .
$$

The following properties are immediate: $\psi(0)>0, \lim _{t \rightarrow \infty} \psi(t)>0$ and the derivative of $\psi$ is increasing on $[0, \infty)$. If we take $p:=\frac{h}{x}$ and $q:=n h$ we have

$$
c_{l}=\frac{n^{l}}{l !} e^{-n x}(x)^{l} \psi(l) .
$$

Then $c_{0}>0$ and $\lim _{l \rightarrow \infty} c_{l}>0$. From (11) it follows that there is $l, 0<$ $l<\infty$ such that $c_{l}<0$. Hence there exists $t_{0} \in(0, \infty)$ such that $\psi^{\prime}(t) \leq 0$, 
$t \in\left[0, t_{0}\right]$ and $\psi^{\prime}(t) \geq 0, t \in\left[t_{0}, \infty\right)$. From these it follows that there is the decomposition $\mathbb{N} \cup\{0\}=I \cup J \cup K$ such that $I<J<K$ and relation (7) holds.

Then by using the well-known relation $S_{n}\left(e_{2}\right)(x)=x^{2}+\frac{x}{n}$ we deduce $\Delta_{h}^{2} S_{n}\left(e_{2}\right)(x)=2 h^{2}$, for any $n \in \mathbb{N}$ and $0<h \leq x$.

Moreover, let $x>0$ and $h>0$ such that $x-h \geq 0$. For each $k \in \mathbb{N} \cup\{0\}$ we have $\left(s_{n, k}\right)^{\prime}=n\left(s_{n, k-1}-s_{n, k}\right)$. We have

$$
\begin{aligned}
\sum_{k=0}^{\infty}\left|s_{n, k}(x+h)-s_{n, k}(x)\right| & \leq \sum_{k=0}^{\infty} \int_{x}^{x+h}\left|\left(s_{n, k}\right)^{\prime}(t)\right| d t \\
& =\sum_{k=0}^{\infty} \int_{x}^{x+h}\left|n\left(s_{n, k-1}-s_{n, k}\right)\right| d t \\
& \leq \sum_{k=0}^{\infty} \int_{x}^{x+h} n s_{n, k}(t) d t+\sum_{k=1}^{\infty} \int_{x}^{x+h} n s_{n, k-1}(t) d t \\
& =\int_{x}^{x+h} \sum_{k=0}^{\infty} n s_{n, k}(t) d t+\int_{x}^{x+h} \sum_{k=1}^{\infty} n s_{n, k-1}(t) d t \\
& =2 n h .
\end{aligned}
$$

In a similar way we have $\sum_{k=0}^{\infty}\left|s_{n, k}(x-h)-s_{n, k}(x)\right| \leq 2 n h$.

Hence $\sum_{k=0}^{\infty}\left|\Delta_{h}^{2} s_{n, k}(x)\right| \leq 4 n h$, i.e. condition (18) fulfills. Consequently, from Theorem 2.2 we have:

Theorem 3.1. Let $f:[0, \infty) \rightarrow \mathbb{R}$ in the domain of $S_{n}, n \in \mathbb{N}$ and let $\rho>0$, such that $\omega_{2}(f, \rho)<\infty$. Then we have

$$
\omega_{2}\left(S_{n}(f), \rho\right) \leq 3 \omega_{2}(f, \rho) .
$$

Also, if $f$ is not a linear function, we have

$$
\limsup _{\rho \rightarrow 0+} \frac{\omega_{2}\left(S_{n}(f), \rho\right)}{\omega_{2}(f, \rho)} \leq 1 .
$$

The same method can be applied to other modified Mirakjan-Favard-Szász operators which preserve linear functions, like the operators defined in [10] or [9]. However for the modified Mirakjan-Favard-Szász operators given in [5] the method may be applied only for the particular case when the function $\varphi$ coincides with the exponential function (see formula (1), given there). 


\section{Baskakov operators}

The Baskakov operators are given by

$$
V_{n}(f)(x)=\sum_{k=0}^{\infty} v_{n, k}(x) f\left(\frac{k}{n}\right), n \in \mathbb{N}, x \in[0, \infty),
$$

where

$$
v_{n, k}(x)=\left(\begin{array}{c}
n+k-1 \\
k
\end{array}\right) x^{k}(1+x)^{-n-k}
$$

and $f:[0, \infty) \rightarrow \mathbb{R}$ is such that series (24) converges.

The operators $V_{n}$ preserve linear functions. It remains to show that condition (7) is satified for certain sets $I<J<K$, when $x-h \in[0, \infty), h>0$. For $p, q \in(0,1) p>q$ and $n \in \mathbb{N}$ we consider the function

$$
\psi(t):=\left(\frac{1+p}{1+q}\right)^{t}(1+q)^{-n}+\left(\frac{1-p}{1-q}\right)^{t}(1-q)^{-n}-2, t \in[0, \infty) .
$$

The following properties are immediate: $\psi(0)=(1+q)^{-n}+(1-q)^{-n}-$ $2>2 \sqrt{\left(1-q^{2}\right)^{-n}}-2>0, \lim _{t \rightarrow \infty} \psi(t)>0\left(\right.$ since $\frac{1+p}{1+q}>1$ and $\left.\frac{1-p}{1-q} \in(0,1)\right)$ and the derivative of $\psi$ is increasing on $[0, \infty)$. If we take $p:=\frac{h}{x}$ and $q:=\frac{h}{1+x}$ we have

$$
c_{l}=\left(\begin{array}{c}
n+l-1 \\
l
\end{array}\right)(x)^{l}(1+x)^{-n-l} \psi(l) .
$$

Then $c_{0}>0$ and $\lim _{l \rightarrow \infty} c_{l}>0$. From (11) it follows that there is $l, 0<$ $l<\infty$ such that $c_{l}<0$. Hence there exists $t_{0} \in(0, \infty)$ such that $\psi^{\prime}(t) \leq 0$, $t \in\left[0, t_{0}\right]$ and $\psi^{\prime}(t) \geq 0, t \in\left[t_{0}, \infty\right)$. From these it follows that there is the decomposition $\mathbb{N} \cup\{0\}=I \cup J \cup K$ such that $I<J<K$ and relation (7) is true.

It is well-known that $V_{n}\left(e_{2}\right)(x)=x^{2}+\frac{x(1+x)}{n}$. Then $\Delta_{h}^{2} V_{n}\left(e_{2}\right)(x)=$ $2 h^{2} \frac{n+1}{n}$, for all $n \in \mathbb{N}$ and $0<h \leq x$.

On the other hand we have $\left(v_{n, k}\right)^{\prime}=n\left(v_{n+1, k-1}-v_{n+1, k}\right)$. Using the same method as for Mirakjan-Favard-Szász operators we obtain $\sum_{k=0}^{\infty}\left|\Delta_{h}^{2} v_{n, k}(x)\right| \leq$ $4 n h$, i.e condition (18) is satisfied.

Then we deduce from Theorem 2.2:

Theorem 3.2. Let $f:[0, \infty) \rightarrow \mathbb{R}$ in the domain of $V_{n}, n \in \mathbb{N}$ and let $\rho>0$, such that $\omega_{2}(f, \rho)<\infty$. Then we have

$$
\omega_{2}\left(V_{n}(f), \rho\right) \leq \frac{3 n+1}{n} \omega_{2}(f, \rho) .
$$


Also, if $f$ is not a linear function, we have

$$
\limsup _{\rho \rightarrow 0+} \frac{\omega_{2}\left(V_{n}(f), \rho\right)}{\omega_{2}(f, \rho)} \leq \frac{n+1}{n} .
$$

\section{References}

[1] J. Adell and A. Pérez-Palomares, Global smothness preservation for the Bernstein polynomials, Approximation and optimization (Proc. Int. Conf. Approximation and Optimization, Cluj-Napoca 1996, ed. by D.D. Stancu et al.), vol I, Cluj-Napoca: Transilvania Press,(1997).

[2] C. Cottin and H. H. Gonska, Simultaneous approximation and global smoothness preservation, Rend. Circ. Mat. Palermo (2) Suppl. Vol. 33, (1993), 259-279.

[3] T. Lindvall, Bernstein polynomials and the law of large numbers, Math. Scientist, Vol. 7, (1982), 127-139.

[4] M. Marin, G. Stan, Weak solutions in Elasticity of dipolar bodies with stretch, Carpathian Journal of Mathematics, Vol. 29 (1), 2013, pp. 33-40.

[5] C. Mortici, An extension of the Szász-Mirakjan operators, An. Şt. Univ. Ovidius Constanța, Vol 17 (1), (2009), 137-144.

[6] R. Păltănea, Best constant in estimates with second order moduli of continuity, In: Approximation Theory, (Proc. Int. Dortmund Meeting on Approximation Theory 1995, ed. by M.W. Müller, M. Felten, D.H. Mache), Berlin: Akad Verlag (1995), 251-275.

[7] R. Păltănea, On the transformation of the second order modulus by Bernstein operators, L'Analyse Numér. et la Th. de l'Approx., Vol. 27 (2), (1998), 309-313.

[8] R. Păltănea, Approximation theory using positive linear operators, Birkh auser, Boston (2004).

[9] R. Păltănea, Modified Szász-Mirakjan operators of integral form, Carpathian Journal of Mathematics, Vol. 24 (3), (2008), 378-385.

[10] R. S. Phillips, An inversion formula for Laplace transforms and semigroups of operators, Annals of Mathematics, Second Series, Vol. 59, (1954), 325-356 
[11] D. X. Zhou, On a problem of Gonska, Results Math., Vol. 28 (1-2), (1995), 169-183.

Radu PĂLTĂNEA,

Transilvania University of Braşov,

Department of Mathematics,

RO-500036 Braşov, Romania.

Email: radupaltanea@yahoo.com

Gabriel STAN,

Department of Mathematics,

Transilvania University of Braşov,

RO-500036 Braşov, Romania.

Email: gabriel.stan@unitbv.ro 